\title{
ARTI APPLICATE, ARTI INDUSTRIALI: UNA QUESTIONE STRATEGICA
}

\author{
ANTONELLO NEGRI (*)
}

SunTO. - Nel progetto di Expo 2015 ha parte importante - al di là del tema specifico - l'idea di una qualità della vita basata su alti requisiti artigianali-manifatturieri nelle loro interazioni con una società industrializzata. Dopo significativi antecedenti ottocenteschi, a Milano è nel primo dopoguerra che si pone la questione strategica di un' "arte sociale", secondo la definizione legata all'esperienza della "sezione artistica" del Partito operaio del Belgio, concretizzatasi nell'attività della Casa del popolo di Bruxelles. La ricerca di migliori standard di vita collettivi sostenuti da un concreto impegno nel miglioramento dell'edilizia popolare e del sistema delle arti applicate trova espressione in una serie di manifestazioni espositive che cominciano a Milano con l'Esposizione regionale lombarda d'arte decorativa della Società Umanitaria del 1919 (contemporanea al Bauhaus di Weimar), continuano con le Biennali di arte decorativa di Monza (1923-1930) e ritornano a Milano nel 1933 con la V Triennale, vetrina della progettazione architettonica, delle arti industriali e della produzione artistica più innovative, che assume subito un respiro internazionale. Tali tematiche continuano ancora oggi a essere il motore propulsore dell'attività della Triennale milanese.

$* * *$

AвSTRACT. - The idea of a quality of life based on high handcraft-manufacturing requirements in their interaction with an industrialised society has an important part beyond the particular case - in the Expo 2015 project. It's in the post-war period that in Milan the strategic question of a "social art" is raised, as defined by the experience of the artistic section of the Belgium Labor Party, put into practice in the activity of the Brussels People's House. The research of better living collective standards sustained by a concrete commitment in the improvement of the social housing and the applied art system is expressed by a series of exhibitions. In Milan with the Regional Lombard Exhibition of decorative arts of the Human Society in 1919 (contemporary to Bauhaus in Weimar) and later on with the Biennial of decorative arts in Monza (1923-1930) and again in Milan in 1933 with the 5th Triennial (showcase of urban planning, industrial arts and most innovative artistic productions) that acquires immediately a world level. These issues are still today the driving force of the Milanese Triennial activity.

${ }^{* *}$ Istituto Lombardo, Università degli Studi di Milano, Italia.

E-mail: antonello.negri@unimi.it 
Nel progetto di Expo 2015 una parte significativa - al di là del tema specifico - è costituita da un'idea di qualità della vita basata anche sulla cosiddetta 'creatività' italiana, di cui fa parte un'antica tradizione di qualità artigiana e manifatturiera che nell'ultimo secolo e mezzo, almeno, ha dovuto fare i conti con una società industrializzata e di massa, come in gran parte d'Europa. Così, la questione della produzione e distribuzione di manufatti su grande scala è diventata anche in Italia una questione strategica, che nei suoi momenti più alti si è manifestata attraverso una specifica ricerca di qualità estetica per i manufatti stessi: dal cucchiaio alla città, per riprendere il famoso slogan coniato nel 1952 dall'architetto Ernesto Nathan Rogers, progettista della milanese Torre Velasca con lo studio BBPR. Negli anni tra le due guerre palcoscenico di tale ricerca di qualità unita alla serialità dell'oggetto prodotto è stata, a Milano (e dintorni, poiché anche Monza è coinvolta), una serie di esposizioni.

La collega Ornella Selvafolta ha concluso il suo intervento parlando della casa popolare come tema di rilievo tra le iniziative della Società Umanitaria. Vorrei ripartire da qui, facendo tre piccoli passi indietro, uno dei quali ci porta fuori Milano, a Torino, e l'altro addirittura a Bruxelles, prima di considerare quanto sarebbe successo a Milano tra 1919 e 1940.

L'inizio ancora molto aurorale di una strategia fortemente milanese nell'ambito della casa popolare va fatto risalire almeno al 1862, subito dopo l'Unità, quando l'idea di ridisegnare la vita degli uomini nella città in via di modernizzazione si concretizzava nell'iniziativa della Società Edificatrice di Case per operaj di bagni e lavatoj pubblici in Milano, che realizzò un grande fabbricato tra le vie San Fermo, Moscova, San Marco e Montebello, ancora esistente ${ }^{1}$. Tale fabbricato costituisce un monumento dimenticato, presto avvertito - allora - come minaccia all'ordine costituito, un po' come sarebbero effettivamente stati gli Höfe della Vienna rossa nel primo dopoguerra². Fu un fatto di

1 Cfr. Relazione annuale del Consiglio d'amministrazione della Società edificatrice di case per gli operaj, bagni e lavatoi pubblici. Adunanza generale dei soci 26 aprile 1863, Milano, A. Lombardi, 1863; G. Gaggi, La Società edificatrice di case per operai, bagni e lavatoi pubblici di Milano. Origine e sviluppo, Milano, Artigianelli, 1935.

2 Cfr. Vienna rossa. La politica residenziale nella Vienna socialista, 1919-1933, a cura di M. Tafuri, Milano, Electa, 1980; E. Blau, The architecture of red Vienna, 19191934, Cambridge, Mass., The MIT Press, 1999. 
grande rilievo che coincise con l'inizio di una strategia, in campo edilizio, di riforme in chiave umanitaria e socialista della città, così forte e gradualmente radicata nel tessuto urbano da andare al di là delle ideologie, se si considera che la questione si sarebbe continuata a porre anche negli anni del fascismo, principalmente - considerato il tema di questo convegno (dunque mettendo da parte tutta la componente dell'edilizia dell'Istituto delle case popolari) - nelle Esposizioni Triennali: del $1933^{3}$ e, soprattutto, del $1936^{4}$, ma senza dimenticare precedenti esposizioni specificamente destinate alle arti applicate e all'architettura, dall'Umanitaria nel $1919^{5}$ alle Biennali e poi Triennali monzesi degli anni Venti ${ }^{6}$. Nel loro insieme tali mostre raccontano il progetto di una strategia di concreta trasformazione della vita degli uomini - anticipatrice dello slogan prima ricordato - sotto il segno dell'utopia di una possibile fruizione della qualità artistica e architettonica per tutti, cui era ormai saldamente ancorata l'idea della riproducibilità industriale delle cose (il design) e delle immagini (fotografia e cinema).

Un secondo passo indietro è relativo al programma di "arte sociale" in un contesto che sarebbe stato di grande influenza per tutta l'Europa: la "sezione artistica" del Partito operaio del Belgio, della quale fu geniale protagonista Henry van de Velde (dal 1902 direttore della Scuola di arti applicate di Weimar da cui sarebbe nato il Bauhaus). Suo centro operativo era la Casa del popolo progettata da Victor Horta - capolavoro del modernismo, sciaguratamente distrutto - dove a cavallo del Novecento si susseguivano manifestazioni artistiche, letterarie e musicali destinate alla classe lavoratrice di Bruxelles ${ }^{7}$. "Arte sociale" voleva anche dire aprirsi

\footnotetext{
3 Cfr. A. Pica, V Triennale di Milano. Catalogo ufficiale, Milano, Ceschina, 1933.

4 Cfr. A. Pica, Guida alla VI Triennale, Milano, Ceschina, 1936.

5 Cfr. Società Umanitaria, Milano, Esposizione regionale lombarda d'arte decorativa, 5 settembre-15 ottobre 1919, Milano, Cooperativa Grafica Operai, 1919; Università delle arti decorative del consorzio Milano Monza Umanitaria retta dalla Società umanitaria nella Villa Reale di Monza, Milano, Cooperativa grafica degli operai, 1922.

6 Cfr. Consorzio autonomo Milano Monza Umanitaria, 1. Mostra biennale delle arti decorative internazionali: maggio-ottobre 1923, Bergamo, Officine dell'Istituto italiano d'arti grafiche, Bergamo 1922. Su questa e sulle successive esposizioni monzesi $(1925,1927,1930)$ e milanesi $(1933,1936,1940)$ si vedano - oltre ai cataloghi delle stesse - A. Pica, Storia della Triennale 1918-1957, Milano Edizioni del Milione, 1957; A. Pansera, Storia e cronaca della Triennale, Milano, Longanesi, 1978 [I Marmi, 92].

7 Cfr. C. Azimonti, La maison du peuple de Bruxelles, Tipografia Paolo Pellegatta, Busto Arsizio, 1920; Le soixantenaire d'Emile Vandervelde. Compte-rendu de
} 
alle arti decorative e applicate - ceramiche, vetri e pannelli decorativi, mobili, libri illustrati e rilegature di libri - la cui rinnovata progettazione e diffusione poteva mettere a disposizione di tutti oggetti esteticamente gradevoli nella loro "modernità" e al tempo stesso portatori di migliori standard di vita per la propria funzionalità. L'importanza data all'arte del libro si riflette altresì nel fatto che libri a stampa erano esposti - senza distinzioni gerarchiche - insieme a pitture e sculture, disegni, incisioni e prodotti di arte decorativa e industriale, a sottolineare l'ampiezza onnicomprensiva degli ambiti d'applicazione di una ricerca e di una produzione da considerarsi a tutti gli effetti d'avanguardia .

Un terzo passo indietro riguarda la Prima esposizione internazionale d'arte decorativa moderna, la prima del genere in Italia, tenutasi a Torino nel 1902. Vi erano compresi i padiglioni dedicati a due nuove forme artistiche basate sulla riproducibilità dell'immagine, fotografia e cinema, mentre negli ambienti integralmente progettati negli spazi dei diversi paesi partecipanti emergeva l'idea della standardizzazione di oggetti e manufatti. Anche in tale occasione l'intreccio di artigianato e invenzione artistica si manifestava nell'arte del libro, come nel caso esemplare della copertina di Also sprach Zarathustra di Nietzsche disegnata dall'architetto Behrens. Filo conduttore primario era comunque quello dello spazio abitativo e di quanto la casa doveva contenere (mobili e soprammobili, carte da parati, tappeti) senza dimenticare il 'contenitore' di tutto, cioè la città'.

Ma torniamo a Milano, che dall'immediato dopoguerra comincia a diventare, in tale prospettiva, il grande punto di riferimento nazionale, a cominciare dalla Esposizione regionale lombarda d'arte decorativa

la fête intime organisée a la Maison du peuple de Bruxelles le 25 janvier 1926, Bruxelles, L'Eglantine, 1926; F. Borsi, La Maison du Peuple. Sindacalismo come arte, Bari, Dedalo, 1978; J. Delhaye, La maison du peuple de Victor Horta, Bruxelles, Atalier Vokaer, 1987.

8 Cfr. Evocation des 20 et de la Libre Esthétique, (Bruxelles, Musées royaux des beaux-arts de Belgique, 29 apr.-10 lug. 1966), Bruxelles, Laconti, 1966; Les XX and the Belgian Avant-Garde: Prints, Drawings, and Books ca. 1890, a cura di S.H. Goddard, The University of Kansas, Spencer Museum of Art, 1992; Les 20 \& la libre esthétique: cent ans aprés, Bruxelles, Musée royal des beaux-arts de Belgique, 1993.

9 Cfr. Prima Esposizione Internazionale d'Arte Decorativa Moderna, Torino 1902, Catalogo Generale Ufficiale, Torino 1902; Torino 1902. Le arti decorative internazionali del nuovo secolo, a cura di R. Bossaglia, E. Godoli, M. Rosci, Milano, Fabbri, 1994; V. Garuzzo, L’Esposizione del 1902 a Torino, Torino, Testo \& Immagine, 1999. 
organizzata dalla Società Umanitaria nel settembre-ottobre $1919^{10}$. Nello stesso anno, può essere utile ricordarlo, in Germania, a Weimar, nasceva il Bauhaus, diretto dall'architetto Walter Gropius ${ }^{11}$.

Tra le idee dei promotori della mostra milanese - Raffaello Bertieri, Giannino Castiglioni, Guido Marangoni, Alfredo Melani, Margherita Sarfatti, per ricordarne alcuni - c'era quella di "incoraggiare la creazione e la produzione di arredi economici per la casa del popolo, che pure deve essere allietata da semplici ma pure forme di bellez$\mathrm{za}^{\prime 12}$. In sé, gran parte dei pezzi esposti era ancora fortemente imbevuta di un gusto tradizionale. D'altra parte le aperture al nuovo non erano poche, al di là di consolidate eccellenze come i ferri di Mazzucotelli: basti citare - oltre alla cucina-sala da pranzo e alla stanza da letto per casa popolare di E. Dozio, estremamente semplici e lineari - le stoffe di Rosa Menni, il protodesign 'ricamato' di Marcello Nizzoli, i giocattoli di carta di E. Altara, le bambole e i giochi di A.B. D'Anna e G. Pero.

D'altra parte, la rinnovata idea di "arredi economici" avrebbe trovato in quel primo dopoguerra una concreta occasione applicativa nei progetti del Comune che proprio dal 1919 cominciavano a concretizzarsi dei quartieri popolari Vittoria, Friuli, Monza, Solari, Giambologna, "alla Fontana", dei cui arredi esiste ancora una qualche pur frammentaria documentazione fotografica negli archivi del vecchio Istituto delle case popolari ${ }^{13}$.

La mostra del 1919 doveva essere la prima di una serie di Esposizioni speciali d'Arte decorativa mirante a "stimolare le genialità creatrici del nostro paese, a educare il gusto del pubblico e, soprattutto, [...] diffondere il bisogno delle cose belle, anche se utili, nel popolo, offrendo anche all'industria produttrice modelli di lavoro, pratici e di buon gusto" ${ }^{14}$. Il tema specifico era l'arredamento della casa, con una

10 Cfr. Esposizione regionale lombarda d'arte decorativa, cit.

11 Dopo Bauhaus 1919-1928, a cura di H. Bayer, W. Gropius, I. Gropius, Teufen, Arthur Niggli, 1938; si vedano, tra le numerosissime pubblicazioni sul tema, H.M. Wingler, Das Baubaus, Rasch-DuMont Schauberg, Bramsche 1962 (trad. it. Feltrinelli, Milano, 1972); M. Droste, Baubaus 1919-1933, Köln, Taschen, 1991; R.K. Wick, Baubaus. Kunstschule der Moderne, Ostfildern-Ruit, Hatje Cantz; 2000; Baubaus Weimar, a cura di M. Siebenbrodt, Milano, Electa, 2008.

12 Esposizione regionale lombarda d'arte decorativa, cit.

13 Cfr. A. Negri, Il sogno del moderno. Architettura e produzione a Milano tra le due guerre, Firenze, Edifir, 1994.

14 Esposizione regionale lombarda d'arte decorativa, cit. 
particolare attenzione per la "casa popolare", cui erano chiamati a concorrere, con idee e progetti, artisti e "artefici" del mobile, delle tappezzerie, delle decorazioni murali, della ceramica, del ferro battuto, dell'argenteria e dei cuoi. Si auspicava allora - come sarebbe successo in Germania con il Bauhaus - che i migliori prototipi potessero entrare nel ciclo della riproduzione industriale, stabilendo un circolo virtuoso tra invenzione artistica e circolazione dei prodotti, nella prospettiva di una sorta di democratizzazione della bellezza.

Era l'inizio di una stagione milanese molto produttiva, da questo punto di vista, il cui snodo fondamentale sarebbe stato l'inaugurazione, nel 1933, della quinta Triennale nel Palazzo dell'Arte appositamente costruito per contenerla ${ }^{15}$. Nel 1923, intanto, la "regionale" del 1919 si era trasformata nella Prima biennale nazionale di arte decorativa nella Villa Reale di Monza (promossa dal Consorzio Milano-MonzaUmanitaria), dove ritroviamo quei temi sociali già tipici della rassegna del $1919^{16}$. Nelle successive tre edizioni monzesi, l'ultima delle quali diventata 'triennale' prima del ritorno a Milano del 1933, si assiste a un progressivo allineamento della ricerca progettuale italiana ai più avanzati standard europei. Se nel $1925^{17}$ è ancora marcata la distanza tra il razionalismo d'impronta già internazionale - esemplificato dalla sezione del Belgio - e, per esempio, la comunque sorprendente visionarietà modernista dei disegni e delle maquette presentati dal milanese Nucleo pittori e architetti (coincidente con la figura di Giandante X) ${ }^{18}$, nelle edizioni successive tale divario si riduce considerevolmente. Basti pensare, per il 1927, al primo gruppo di architetti razionalisti italiani, il

15 Cfr. O. Hoffmann, Le strutture in cemento armato del Palazzo dell'arte di Milano, in "Rassegna di architettura", V, 5,15 maggio 1933; Giovanni Muzio. Il Palazzo dell'arte. Disegni e progetto del Palazzo dell'arte, a cura di L. Fiori e M.P. Belski, Milano, Abitare Segesta, 1982; F. Irace, Giovanni Muzio. Opere, Milano, Electa, 1995.

16 Cfr. 1. Mostra biennale delle arti decorative internazionali, cit.

17 Cfr. Seconda Mostra internazionale delle arti decorative. Consorzio MilanoMonza Umanitaria, maggio-ottobre 1925, Milano, Alpes, 1925.

18 Cfr. G. Traversi, Giandante X. Vita e arte, Milano, G. Caruso, 1963; A. Negri, S. Robertazzi, Giandante X, l'avanguardia dimenticata, Milano, Fondazione Corrente, 1988; A. Negri, Giandante X (1899-1984). Ricerca artistica pura e decorazione d'agitazione, in "Studi di storia delle arti", 1991 (6), 209-227; R. Farina, Giandante X, Milano, Milano 2014; A. Capozza, Giandante X artista della libertà. Dalla guerra civile di Spagna ai campi di concentramento di Francia, alla Resistenza in Italia, Milano, www.aicvas.org, 2014. 
Gruppo 7, che a Monza espone i suoi modelli e i suoi progetti, fra i quali una Officina del gas di Giuseppe Terragni e il Dopolavoro di Luigi Figini e Gino Pollini; il padiglione del libro vi era stato disegnato da Fortunato Depero, mentre l'architetto Giovanni Muzio e il pittore Mario Sironi avevano ideato una Mostra grafica d'impostazione estremamente avanzata ${ }^{19}$. Quanto alla Quarta Triennale Internazionale di arte decorativa del 1930, va ricordato in primo luogo quell'esemplare modello di razionalismo e di moderna funzionalità standardizzabile costituito dalla casa elettrica di Figini, Frette, Libera, Pollini e Bottoni, costruita nel parco di Monza ${ }^{20}$.

Il trasferimento della Triennale di Monza a Milano avviene, nel 1933, nel quadro di un'esplicita riorganizzazione, da parte del regime fascista, del complessivo sistema espositivo italiano, che comprende anche la Biennale internazionale di Venezia e la Quadriennale nazionale di Roma ${ }^{21}$. A guidare la Triennale è adesso un Direttorio costituito da Mario Sironi, Gio Ponti, Giulio Barella e Carlo Alberto Felice 22. L'importanza anche politica della prima Triennale milanese - quinta della serie - è tra l'altro attestata da una presenza delle alte gerarchie fasciste e della famiglia reale all'inaugurazione con gran risonanza pubblica nei mezzi di comunicazione di massa, giornali e copertine di riviste illustrate, a dimostrare il rilievo che s'intendeva dare alla ritualità di tali rinnovate manifestazioni, nell'economia di un nuovo sistema dell'arte organizzato e controllato capillarmente dal regime ${ }^{23}$. La Triennale,

19 Catalogo ufficiale della $3^{a}$ Mostra internazionale delle arti decorative, maggioottobre 1927, Monza, Milano, Ceschina, 1927.

20 Cfr. Catalogo ufficiale della IV Esposizione triennale internazionale delle arti decorative ed industriali moderne, Milano, Ceschina, 1930. Oltre ai cataloghi delle esposizioni monzesi si veda - per il quadro dell'educazione artistica a Monza - L'ISIA a Monza. Una scuola d'arte europea, a cura di R. Bossaglia, Monza, Associazione Pro Monza, 1986.

21 Cfr. S. Salvagnini, Il sistema delle arti in Italia, 1919-1943, Bologna, Minerva, 2000.

22 Cfr. S. Bignami, 1933. Un anno del Novecento a Milano, Milano, Skira, 2001; E. Longari, Sironi e la $5^{a}$ Triennale di Milano, Nuoro, Ilisso, 2007.

23 Cfr. La Triennale, in Anni'30. Arti in Italia oltre il fascismo (Firenze, Palazzo Strozzi, 22 settembre 2012-27 gennaio 2013), a cura di A. Negri con S. Bignami, P. Rusconi, G. Zanchetti, Firenze, Giunti, 2012, 198-203; A. Negri, Opere d'arte e artisti nella stampa periodica fra le due guerre, in Arte moltiplicata. L'immagine del '900 italiano nello specchio dei rotocalchi, a cura di B. Cinelli, F. Fergonzi, M.G. Messina, A. Negri, Milano, Bruno Mondadori, 2013, 1-46. 
d'altra parte, si configurava quale vetrina dell'arte, dell'architettura e del design italiani, destinata anche a un pubblico internazionale.

Tutti i più importanti artisti italiani del tempo vi erano rappresentati: a cominciare dagli scultori Arturo Martini e Marino Marini e dai pittori Mario Sironi, Achille Funi e Giorgio de Chirico, che con i loro grandi murali celebravano l'Italia nelle diverse manifestazioni del lavoro, dello sport, dello studio e della vita famigliare.

Ma la grande affermazione della V Triennale va soprattutto fatta coincidere con la Mostra internazionale di architettura moderna, organizzata nel lungo emiciclo al piano terra del palazzo: essa doveva rappresentare al pubblico uno stile - scriveva Roberto Papini in "Emporium" nel dicembre 1933 - che "ha la sua radice nella tecnica e il suo fiore nella fantasia" ${ }^{24}$.

$\mathrm{Al}$ visitatore si presentava la visione di una spettacolare galleria con accampata al centro una serie di 'fotomosaici' disposti come quinte teatrali, a offrire una panoramica dell'architettura recente di ciascun paese presente all'esposizione. Sui lati, delle gigantografie proponevano le architetture italiane e straniere distribuite per soggetti; in una terza sezione c'erano le opere realizzate dagli architetti italiani negli ultimi anni, mentre una quarta era dedicata alle "più spiccate personalità dell'architettura moderna", come si legge nel catalogo.

Fuori dal palazzo, nel parco, l'allestimento della Triennale era arricchito dalla Mostra dell'abitazione, consistente in oltre trenta dimore tipo, veri e propri padiglioni di architettura. Le case-padiglione, completamente arredate secondo il gusto moderno, erano state affidate a giovani architetti e dedicate a problemi determinati da bisogni particolari: dalla casa di un conduttore di fattoria alla casa per le vacanze di un artista sul lago, dalla casa del sabato per i giovani sposi alla casa interamente realizzata in acciaio, dal gruppo di cinque case per le vacanze alla villa studio per un artista e alla casa popolare. Le diverse tipologie intendevano collaudare nuove ricerche distributive degli spazi in relazione a bisogni e funzionalità diverse, nonché le applicazioni dei materiali moderni, senza alcuna ridondanze decorativa.

Già tre anni prima, in un articolo per "La Casa Bella", il critico Edoardo Persico aveva sostenuto come l'“apparizione serrata e prepo-

24 R. Papini, La quinta Triennale a Milano. Ispezione alle arti, in "Emporium", 468, dicembre $1933,344$. 
tente" dei mobili in ferro rappresentasse "il fatto più importante dell'arredamento degli ultimi anni", ai quali ha imposto "uno stile insolito e originalissimo" 25 . Tale nuovo stile venne applicato nella tipologia della sedia 'tubolare' in metallo e legno (anche compensato): la sua evoluzione - dalla SIAM di Giuseppe Pagano alle seggioline di Giuseppe Terragni per i bambini dell'asilo Sant'Elia di Como, passando attraverso i modelli di Agnoldomenico Pica e Gabriele Mucchi - mostra come la tendenza fosse di dialogare con spazi della vita quotidiana funzionali, razionali, senza orpelli, riprendendo quell'idea di vita e comportamento sachlich - oggettivo, dinamico, efficiente - che dalla Germania degli anni Venti si era irradiata in tutta Europa e oltre ${ }^{26}$. Nella V Triennale questa tipologia di arredo è esemplarmente rappresentata dai banchi e dalle sedie della Mostra della scuola. L'idea implicita era altresì quella dell'arredamento come architettura vera e propria, che vide coinvolti tanti giovani architetti: spesso per pura necessità, cioè per la mancanza di commissioni più importanti, ma soprattutto con l'idea "morale" di contribuire al miglioramento in chiave moderna, attraverso la propria opera, di comportamenti e stili di vita.

Nella Triennale del 1933, le tipologie proposte nella Mostra dell'abitazione erano estremamente eleganti e ancora sostanzialmente esclusive, limitate a una ristretta cerchia di pubblico, pur evidenziando un'inclinazione già esplicitamente "moderna" e razionalista. Nell'edizione del 1936 invece - accanto al permanente piacere del pezzo unico, almeno nella Mostra dell'arredamento - emergeva un'idea di serialità e standardizzazione, in realtà ormai affermata e praticata, contraddistinta da una progettazione di spazi e complementi d'arredo teoricamente destinati a un impiego diffuso, se non già di massa (come nella parte notturna di un'abitazione progettata dal gruppo BBPR). Tale aspetto è ancor più marcato nell'edizione del 1940 con la Mostra internazionale della produzione di serie.

Le esposizioni sono state il luogo di sperimentazione di nuove prospettive di comportamenti collettivi, con immediato riscontro nella città nuova, come - concludendo - si può esemplificare attraverso alcuni esempi riguardanti la vita quotidiana, il lavoro e il tempo libero.

Per quanto riguarda il tema dell'abitare, molte ricerche e propo-

25 [E. Persico], Mobili in ferro, in "La Casa Bella", III, 34, ottobre 1930, p.n.n.

26 Cfr. A. Negri, Carne e ferro, Milano, Scalpendi, 2007. 
ste avanzate nelle Triennali di Milano trovarono applicazione, per esempio, nelle case popolari, per operai, standardizzate: "un campo adatto [...] all'applicazione dei concetti razionalisti" per l'eliminazione di ogni "superstruttura decorativa inutile" e l'attuazione del progetto con "mezzi minimi", come si legge già nel 1933 nei documenti dell'Istituto Case Popolari di Milano ${ }^{27}$. Quanti ai luoghi di lavoro, bisogna in primo luogo ricordare il palazzo Montecatini di Gio Ponti, finito nel 1938, la cui organizzazione interna era in grado, grazie a un sistema di ascensori "ultrarapidi" e alla distribuzione degli uffici, standardizzati secondo un modulo tipo, di convogliare 1500 impiegati al posto di lavoro nel tempo massimo di una decina di minuti (e silenziosamente, grazie ai pavimenti ricoperti di linoleum $)^{28}$. Luoghi esemplari della città moderna prefigurata in tanti suoi aspetti, prima nelle esposizioni monzesi e poi nelle Triennali, sono infine i nuovi caffè, tra i quali bisogna in primo luogo ricordare il Caffè-Bar Craja degli architetti Baldessari, Figini e Pollini, caratterizzato da un ambiente interno pensato secondo un rigoroso assetto geometrico di linee e piani ortogonali convergenti nella fontana in metallo nichelato di Fausto Melotti, davanti alla parete di fondo.

È una tipologia, quella del caffè, che - osserva Persico scrivendo della Città che si rinnova in "La Casa Bella" (aprile 1931) - "rappresenta a Milano l'ultima espressione del gusto moderno", dove "... pare che la ruota del tempo debba girare in un'atmosfera di sogni astrali e di splendori incantati" 29 . "Se qualcuno mi invitasse domani al Cova - ci ricorda per parte sua Pietro Maria Bardi - gli direi: no, caro, andiamo al Craja" ${ }^{30}$ : le ragioni addotte sono la paura che gli cada in testa il soffitto, il timore di sorbire bevande decrepite, l'avversione verso i bicchieri non lavati nello sciacquatore elettrico, lo sconcerto d'imbattersi negli amici del nonno e la mancanza di comunicazione dei risultati sportivi.

Fatto sta che il Cova - a differenza del Craja - esiste ancora.

27 Cfr. Negri, Il sogno del moderno, cit.

28 "Casabella", XI, 138-140), giugno-agosto 1939.

29 [E. Persico], "La Casa Bella", IV, 40, aprile 1931, p.n.n.

30 P.M. Bardi, Pianta di Milano. Caffè antichi e nuovi, in "L'Ambrosiano", 23 agosto 1932. 